\title{
La colección de meteoritos del Museo de Ciencias Naturales de Tenerife: catalogación internacional y resultados preliminares
}

\author{
The meteorite collection of the Museo de Ciencias Naturales \\ de Tenerife: international cataloguing and preliminary results
}

\author{
S. Hernández-Fernández ${ }^{1}$, J.A. Rodríguez-Losada ${ }^{1}$, F. García Talavera ${ }^{2}$, \\ R. Lunar-Hernández ${ }^{3}$, J. Martínez-Frías ${ }^{4}$
}

\begin{abstract}
RESUMEN
El Museo de Ciencias Naturales de Tenerife (MCNT), cuenta con una importante colección de meteoritos, recolectados desde el año 1985 en diversas expediciones a la zona Sur de Marruecos, Sahara, Mauritania y Senegal. Se han seleccionado siete ejemplares (lititos), con objeto de estudiarlos y catalogarlos, siguiendo la estructura de las bases de datos internacionales sobre meteoritos (por ej., Museo de Historia Natural, Londres). Se aportan datos descriptivos tales como nomenclatura provisional, localización, tipo de encuentro, número de fragmentos, dimensiones, peso, densidad, mineralogía y geoquímica de las principales fases minerales. Se ha realizado una clasificación preliminar de acuerdo a las normas de «The Meteoritical Society».
\end{abstract}

Palabras clave: Meteoritos, Catalogación, Museo, Tenerife.

\begin{abstract}
The Tenerife Museum of Natural Sciences (MCNT), holds an important collection of meteorites, collected since 1985 in various expeditions to the south of Morocco, Sahara, Mauritania and Senegal. Seven specimens (stones) have been selected for study and cataloguing, following the structure of the international databases on meteorites (e.g., Natural History Museum, London). Descriptive data such as provisional nomenclature, location, type of event, number of fragments, dimensions, weight, density, mineralogy and geochemistry of mineral phases are provided. A previous classification was made in accordance with the rules of The Meteoritical Society.
\end{abstract}

Key words: Meteorites, Catalogue, Museum, Tenerife.

\section{Introducción}

El Museo de Ciencias Naturales de Tenerife (MCNT) perteneciente al Organismo Autónomo de Museos y Centros del Cabildo Insular de Tenerife cuenta con colecciones históricas de gran interés científico. Gracias a las expediciones realizadas desde el año 1985 por investigadores del MCNT a la zona Sur de Marruecos, Sahara, Mauritania y Senegal se ha conseguido reunir un importante con- junto de ejemplares de meteoritos (fig. 1), cuyo estudio y catalogación se ha decidido llevar a cabo, dado que este material, depositado en la litoteca del Museo no ha sido analizado hasta el momento de manera sistemática.

Esta actividad del MCNT se enmarca en la labor que viene realizando en relación con la Geología Planetaria (exposiciones científicas, campañas de investigación de cráteres meteoríticos en Mauritania, conferencias y mesas redondas de divulgación, etc.),

\footnotetext{
1 Dpto. Edafología y Geología. Universidad de La Laguna. 38206 La laguna, Tenerife. Email: jrlosada@ull.es

2 Organismo Autónomo de Museos y Centros. Cabildo de Tenerife. Email: fgarcia@ museosdetenerife.org

3 Dpto. Cristalografía y Mineralogía. Universidad Complutense de Madrid. 28040 Madrid. Email: lunar@geo.ucm.es

4 Centro de Astrobiología CSIC/INTA. Ctra. Ajalvir. 28850 Torrejón de Ardoz. Madrid. Email: martinezfj@inta.es
} 


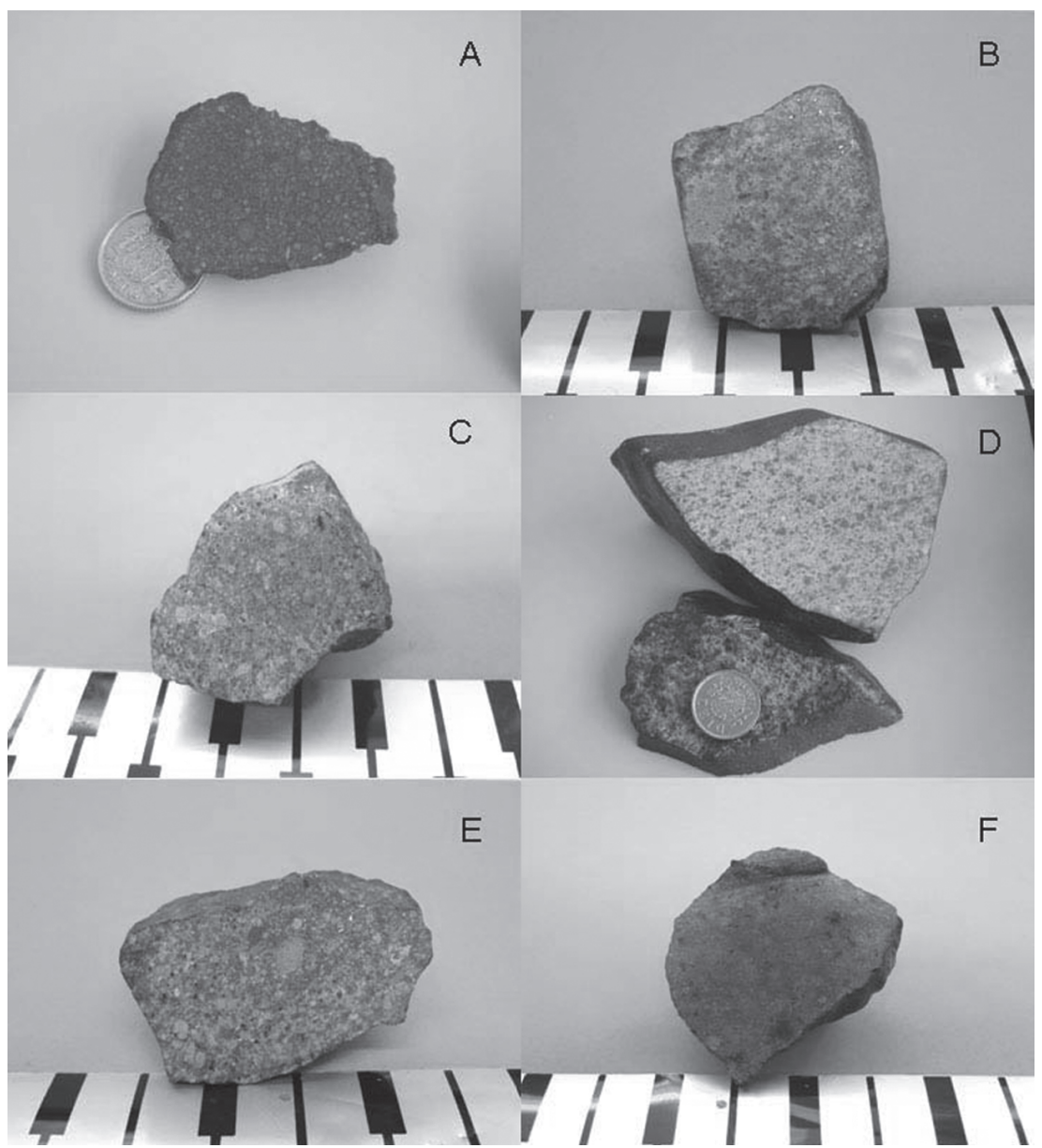

Fig. 1.-Aspecto «de visu» de 6 ejemplares de la colección del MCNT. A) OAMC-1 NWA; B) OAMC-5 NWA; C) OAMC-4 NWA; D) OAMC-2 NWA; E) OAMC-6 NWA; F) OAMC-7 NWA.

en la que participan distintas instituciones y universidades españolas. También es importante subrayar la continuidad de estos trabajos en relación con otros estudios previos realizados por algunos co-autores de la presente contribución, que han abordado, entre otros: la caracterización de distintos tipos de meteoritos asteroidales y de Marte (Martínez-Frías et al., 1989, Rull et al., 2004), la incorporación de nuevos meteoritos españoles al catálogo internacional como el Valencia H5 (Muñoz Sanz, 1997), la actualización del catálogo del Museo Nacional de Ciencias Naturales de Madrid (Muñoz-Espadas et al., 2002) y la contribución en congresos científicos, dando a conocer la colección del Museo de Ciencias Naturales de Tenerife y los avances de los estudios que permitirán su catalogación (Hernández-Fernández et al., 2008).

Inicialmente se han seleccionado siete ejemplares (condritas ordinarias, véase figura 1), cuyo estudio constituye la primera fase de la catalogación que se pretende llevar a cabo como parte de la tesis doctoral titulada: «Catalogación y caracterización de las muestras meteoríticas del Museo de Ciencias Naturales de Tenerife», siguiendo la estructura de las bases de datos internacionales sobre meteoritos como la del Museo de Historia Natural de Londres (Grady, 2000). Para todos ellos se aportan datos acerca de lugar de procedencia en el NW de África (todos ellos son hallazgos), propiedades descriptivas, mineralogía, geoquímica global y detallada de las principales fases minerales (microscopía de luz transmitida y reflejada, DRX, ICP-MS y microsonda electrónica).

Recientemente se han enviado los datos obtenidos hasta el momento del estudio de los siete ejemplares, al Meteoritical Bulletin, con el objeto de obtener una numeración oficial dentro del catálogo y de acuerdo a la normativa de la Meteoritical 
Tabla 1.-Condritas ordinarias

\begin{tabular}{clcl}
\hline Número oficial & \multicolumn{1}{c}{ Lugar } & C/H & \multicolumn{1}{c}{ Clase } \\
\hline OAMC-1 (NWA) & Mahbes (Western Sahara) & $\mathrm{H}$ & Condrita ordinaria tipo 3 \\
OAMC-2 (NWA) & Mahbes (Western Sahara) & $\mathrm{H}$ & Condrita ordinaria (L) \\
OAMC-3 (NWA) & Mahbes (Western Sahara) & $\mathrm{H}$ & Condrita ordinaria (L) \\
OAMC-4 (NWA) & Tirsal (Morocco) & $\mathrm{H}$ & Condrita ordinaria (L) \\
OAMC-5 (NWA) & Tirsal (Morocco) & $\mathrm{H}$ & Condrita ordinaria (L) \\
OAMC-6 (NWA) & Tirsal (Morocco) & $\mathrm{H}$ & Condrita ordinaria (L) \\
OAMC-7 (NWA) & Tirsal (Morocco) & & Condrita ordinaria (LL) \\
\hline
\end{tabular}

C/H (C: Caída, H: hallazgo).

Tabla 2.-Número de fragmentos, dimensión, peso total (Wt) y densidad (d), estado de choque y grado de meteorización, de cada uno de los ejemplares condríticos

\begin{tabular}{|c|c|c|c|c|c|c|}
\hline Número oficial & $\begin{array}{c}\mathrm{N} \mathrm{N}^{\mathrm{o}} \mathrm{de} \\
\text { fragmentos }\end{array}$ & $\begin{array}{l}\text { Dimensiones } \\
(\mathrm{cm})\end{array}$ & $\begin{array}{l}\text { Wt } \\
(\mathrm{g})\end{array}$ & $\begin{array}{c}\mathrm{d} \\
\left(\mathrm{g} / \mathrm{cm}^{3}\right)\end{array}$ & $\begin{array}{c}\text { Estado } \\
\text { de choque }\end{array}$ & $\begin{array}{c}\text { Grado de } \\
\text { meteorización }\end{array}$ \\
\hline OAMC-1 (NWA) & 4 & $4 \times 2,7$ & 8,1 & 3,12 & $\mathrm{~S} 1$ & $\mathrm{~W} 0 / 1$ \\
\hline OAMC-2 (NWA) & 1 & $9,4 \times 6,73$ & 344,3 & 3,41 & $\mathrm{~S} 2$ & $\mathrm{~W} 1 / 2$ \\
\hline OAMC-3 (NWA) & 1 & $15,5 \times 10,5$ & $2.140,2$ & 3,34 & $\mathrm{~S} 2$ & $\mathrm{~W} 1 / 2$ \\
\hline OAMC-4 (NWA) & 1 & $6,9 \times 3,4$ & 103,7 & 3,41 & $\mathrm{~S} 1$ & $\mathrm{~W} 1 / 2$ \\
\hline OAMC-5 (NWA) & 1 & $5,2 \times 3,3$ & 92,5 & 3,41 & S2 & $\mathrm{W} 1 / 2$ \\
\hline OAMC-6 (NWA) & 1 & $5,2 \times 3,9$ & 106,3 & 3,42 & $\mathrm{~S} 2$ & $\mathrm{~W} 1 / 2$ \\
\hline OAMC-7 (NWA) & 1 & $4,3 \times 3,44$ & 73 & 3,38 & S3 & $\mathrm{W} 2 / 3$ \\
\hline
\end{tabular}

Society. El Meteoritical Bulletin contiene el listado de todos los meteoritos reconocidos y clasificados, que son aceptados por el Nomenclature Committee. Por el momento «the Nomenclature Committee de la Meteoritical Society», le ha otorgado a cada una de las muestras de la colección del MCNT, un número provisional dentro del grupo NWA, dado que los ejemplares de estudio provienen del NW de África. Se seguirá usando la denominación inicial hasta que la Meteoritical Society asigne un número oficial.

\section{Meteoritos del MCNT: descripción general y caracterización}

Las tablas 1 y 2 resumen algunas de las características de los meteoritos condríticos del MCNT, denominación asignada por la Meteoritical Society, las áreas geográficas en que fueron encontrados, una primera clasificación en función de los datos obtenidos hasta el momento, así como una serie de propiedades descriptivas de cada uno de los ejemplares estudiados.

En general, las muestras estudiadas presentan una fina corteza de fusión de color gris oscuro, casi negra y unas ondulaciones superficiales muy poco marcadas a modo de regmaglifos, generados por la fricción con la atmósfera. En el corte se suelen apreciar a simple vista los cóndrulos, como esferas oscuras y en algunas de las muestras se aprecian granos metálicos.

\section{Descripción petrográfica}

Todos los ejemplares se caracterizan por la presencia de cóndrulos, constituidos por silicatos (olivino, piroxeno y plagioclasa), metales, óxidos y una estructura interna variable. En todos ellos, la morfología de cóndrulos presenta formas redondeadas o semirredondeadas o bien fragmentados, en algunos casos muy difusos y de difícil distinción. El tamaño de los cóndrulos varía desde 1 a $2 \mathrm{~mm}$ y aparecen englobados en una matriz microcristalina predominantemente de color gris oscuro o con tonalidad marrón. Tanto en cóndrulos como en matriz se distingue olivino, piroxeno, feldespato en uno de los ejemplares. En algunos casos la matriz presenta troilita y aleación de $\mathrm{Fe}-\mathrm{Ni}$ (kamacita dominante) de forma dispersa. En algunos cóndrulos, tanto olivino como piroxeno aparecen en disposición radiada (figs. 2 y 3 ). 

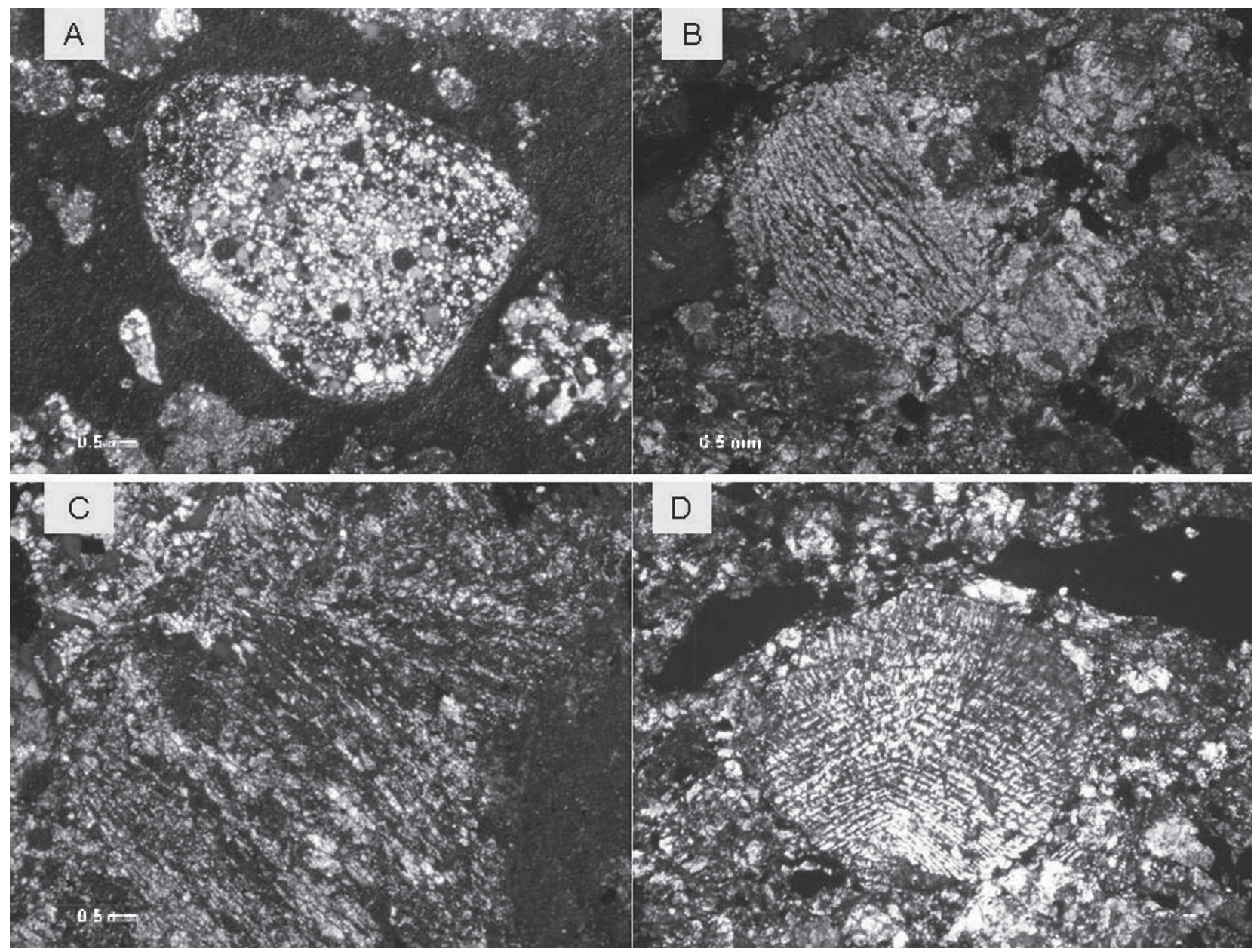

Fig. 2.-Lámina delgada en nícoles cruzados. A) OAMC-1 NWA; B) OAMC-2 NWA; C) OAMC-3 NWA; D) OAMC-4 NWA.

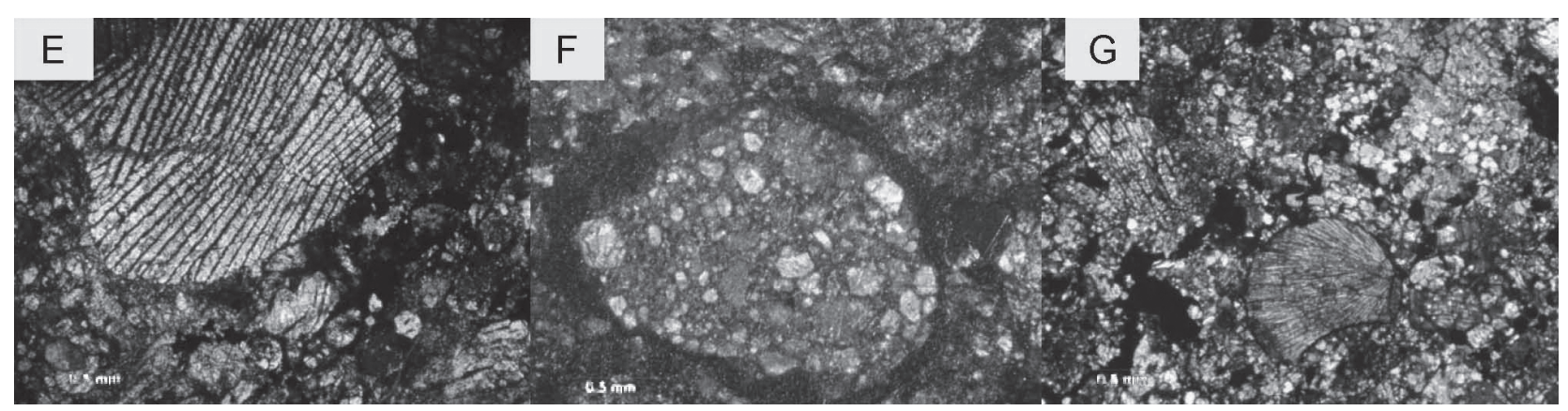

Fig. 3.—Lámina delgada en nícoles cruzados. E) OAMC-5 NWA; F) OAMC-6 NWA; G) OAMC-7 NWA.

\section{Composición de las muestras}

Las principales fases minerales son olivino, piroxeno (principalmente broncita), plagioclasas (albita, oligoclasa), troilita, aleaciones de $\mathrm{Fe}-\mathrm{Ni}$ (kamacita y taenita) y óxidos (cromita, magnetita). Desde el punto de vista geoquímico todos los ejemplares muestran una composición homogénea, sin variaciones significativas de los principales elementos químicos. No obstante, los contenidos en $\mathrm{Fe}$ y $\mathrm{Mg}$ presentan rangos de variación entre $24,47 \%$ (OAMC-7 NWA) y 30,33\% (OAMC-2 NWA), y 



Fig. 4.-Imágenes de microsonda electrónica: sup. izquierda. OAMC-1 NWA; sup. derecha. OAMC-3 NWA; inf. izquierda OAMC-5 NWA; inf. derecha OAMC-6 NWA.

entre $21,65 \%$ (OAMC-1 NWA) y 24,18\% (OAMC3 NWA) respectivamente. Con relación al $\mathrm{Ni}$, todos los resultados se encuentran entre el 1 y el $2 \%$. Específicamente, se han realizado más de 100 análisis de microsonda (fig. 4) cuyos resultados se sintetizan en las tablas 3 a 8 , donde las composiciones de los distintos minerales analizados están agrupadas en distintas tablas correspondientes a cada una de las fases minerales.

\section{Imágenes de microsonda electrónica}

En imágenes obtenidas durante la microsonda electrónica, se pueden observar diferentes tonalidades en función del mineral analizado. Previamente se analiza un mineral mediante microscopio petrográfico y sobre ese mineral identificado se hace el análisis de microsonda electrónica, obteniendo la composición del mineral en cuestión (fig. 4). 
Tabla 3.-Composición de olivinos presentes en las muestras de meteoritos

\begin{tabular}{lrrl}
\hline Meteorito & \multicolumn{2}{l}{ \% Fo } & \multicolumn{2}{l}{ Fórmula } \\
\hline OAMC-1 (NWA) & 91,14 & 8,86 & $\left(\mathrm{Fe}_{0,178} \mathrm{Mg}_{1,830}\right) \mathrm{Si}_{0,985} \mathrm{O}_{4}$ \\
& 98,06 & 1,94 & $\left(\mathrm{Fe}_{0,039} \mathrm{Mg}_{1,976}\right) \mathrm{Si}_{0,988} \mathrm{O}_{4}$ \\
& 73,73 & 26,27 & $\left(\mathrm{Fe}_{0,524} \mathrm{Mg}_{1,471}\right) \mathrm{Si}_{0,996} \mathrm{O}_{4}$ \\
& 98,06 & 1,94 & $\left(\mathrm{Fe}_{0,039} \mathrm{Mg}_{1,976}\right) \mathrm{Si}_{0,988} \mathrm{O}_{4}$ \\
& 98,80 & 1,20 & $\left(\mathrm{Fe}_{0,024} \mathrm{Mg}_{1,983}\right) \mathrm{Si}_{0,989} \mathrm{O}_{4}$ \\
& 99,64 & 0,36 & $\left(\mathrm{Fe}_{0,007} \mathrm{Mg}_{1,961}\right) \mathrm{Si}_{0,991} \mathrm{O}_{4}$ \\
\hline OAMC-2 (NWA) & 75,92 & 24,08 & $\left(\mathrm{Fe}_{0,484} \mathrm{Mg}_{1,526}\right) \mathrm{Si}_{0,986} \mathrm{O}_{4}$ \\
& 75,89 & 24,11 & $\left(\mathrm{Fe}_{0,489} \mathrm{Mg}_{1,539}\right) \mathrm{Si}_{0,981} \mathrm{O}_{4}$ \\
& 75,75 & 24,25 & $\left(\mathrm{Fe}_{0,491} \mathrm{Mg}_{1,534}\right) \mathrm{Si}_{0,981} \mathrm{O}_{4}$ \\
\hline OAMC-3 (NWA) & 75,01 & 24,99 & $\left(\mathrm{Fe}_{0,507} \mathrm{Mg}_{1,522}\right) \mathrm{Si}_{0,979} \mathrm{O}_{4}$ \\
& 74,93 & 25,07 & $\left(\mathrm{Fe}_{0,477} \mathrm{Mg}_{1,426}\right) \mathrm{Si}_{1,038} \mathrm{O}_{4}$ \\
& 72,46 & 27,54 & $\left(\mathrm{Fe}_{0,570} \mathrm{Mg}_{1,500}\right) \mathrm{Si}_{0,958} \mathrm{O}_{4}$ \\
\hline OAMC-5 (NWA) & 78,92 & 21,08 & $\left(\mathrm{Fe}_{0,426} \mathrm{Mg}_{1,595}\right) \mathrm{Si}_{0,980} \mathrm{O}_{4}$ \\
& 78,98 & 21,02 & $\left(\mathrm{Fe}_{0,422} \mathrm{Mg}_{1,586}\right) \mathrm{Si}_{0,989} \mathrm{O}_{4}$ \\
\hline OAMC-6 (NWA) & 76,07 & 23,93 & $\left(\mathrm{Fe}_{0,412} \mathrm{Mg}_{1,310}\right) \mathrm{Si}_{1,109} \mathrm{O}_{4}$ \\
& 75,66 & 24,34 & $\left(\mathrm{Fe}_{0,478} \mathrm{Mg}_{1,486}\right) \mathrm{Si}_{1,010} \mathrm{O}_{4}$ \\
\hline OAMC-7 (NWA) & 79,37 & 20,63 & $\left(\mathrm{Fe}_{0,416} \mathrm{Mg}_{1,600}\right) \mathrm{Si}_{0,987} \mathrm{O}_{4}$ \\
\hline
\end{tabular}

Fo: Forsterita; Fa: Fayalita.

Tabla 4.-Composición de los feldespatos presentes en las muestras de meteoritos

\begin{tabular}{lll}
\hline Meteorito & \multicolumn{1}{c}{ Mineral } & \multicolumn{1}{c}{ Fórmula } \\
\hline OAMC-2 & Oligoclasa & $\mathrm{Ca}_{0,195} \mathrm{Na}_{0,811} \mathrm{Al}_{1,098} \mathrm{Si}_{2,876} \mathrm{O}_{8}$ \\
\cline { 2 - 3 } & $\begin{array}{l}\text { Albita- } \\
\text { Oligoclasa- }\end{array}$ & $\mathrm{K}_{0,102} \mathrm{Ca}_{0,109} \mathrm{Na}_{0,806} \mathrm{Al}_{1,112} \mathrm{Si}_{2,872} \mathrm{O}_{8}$ \\
& $\begin{array}{l}\text { Anortosa } \\
\end{array}$ & \\
& $\begin{array}{l}\text { Albita- } \\
\text { Oligoclasa }\end{array}$ & $\mathrm{Ca}_{0,105} \mathrm{Na}_{0,8763} \mathrm{Al}_{1,085} \mathrm{Si}_{2,907} \mathrm{O}_{8}$ \\
\hline
\end{tabular}

Tabla 5.-Composición de las aleaciones Fe-Ni presentes en las muestras de meteoritos

\begin{tabular}{cll}
\hline \multicolumn{1}{c}{ Meteorito } & Mineral & \multicolumn{1}{c}{ Fórmula } \\
\hline OAMC-2 (NWA) & Kamacita & $\mathrm{Fe}(93,27 \%) ; \mathrm{Ni}(6,73 \%)$ \\
& Kamacita & $\mathrm{Fe}(93,48 \%) ; \mathrm{Ni}(6,52 \%)$ \\
& Kamacita & $\mathrm{Fe}(94,97 \%) ; \mathrm{Ni}(5,03 \%)$ \\
& Kamacita & $\mathrm{Fe}(94,22 \%) ; \mathrm{Ni}(5,78 \%)$ \\
& Kamacita & $\mathrm{Fe}(93,75 \%) ; \mathrm{Ni}(6,25 \%)$ \\
\hline OAMC-5 (NWA) & Taenita & $\mathrm{Fe}(50,66 \%) ; \mathrm{Ni}(49,34 \%)$ \\
& Kamacita & $\mathrm{Fe}(94,89 \%) ; \mathrm{Ni}(5,11 \%)$ \\
& Kamacita & $\mathrm{Fe}(92,02 \%) ; \mathrm{Ni}(7,98 \%)$ \\
& Kamacita & $\mathrm{Fe}(93,09 \%) ; \mathrm{Ni}(6,91 \%)$ \\
& Taenita & $\mathrm{Fe}(59,72 \%) ; \mathrm{Ni}(40,28 \%)$ \\
& Kamacita & $\mathrm{Fe}(95,06 \%) ; \mathrm{Ni}(4,94 \%)$ \\
\hline OAMC-6 (NWA) & Taenita & $\mathrm{Fe}(79,66 \%) ; \mathrm{Ni}(20,34 \%)$ \\
& Taenita & $\mathrm{Fe}(73,19 \%) ; \mathrm{Ni}(26,81 \%)$ \\
& Kamacita & $\mathrm{Fe}(94,22 \%) ; \mathrm{Ni}(5,78 \%)$ \\
\hline
\end{tabular}

Tabla 6.-Composición de los piroxenos presentes en las muestras de meteoritos

\begin{tabular}{lll}
\hline Meteorito & Mineral & \multicolumn{1}{c}{ Fórmula } \\
\hline $\begin{array}{l}\text { OAMC-1 } \\
\text { (NWA) }\end{array}$ & Enstatita & $\left(\mathrm{Mg}_{0,959} \mathrm{Fe}_{0,027}\right) \mathrm{Si}_{0,991} \mathrm{O}_{3}$ \\
\hline $\begin{array}{l}\text { OAMC-2 } \\
\text { (NWA) }\end{array}$ & Broncita & $\left(\mathrm{Mg}_{0,788} \mathrm{Fe}_{0,195}\right) \mathrm{SiO}_{3}$ \\
\hline $\begin{array}{l}\text { OAMC-3 } \\
\text { (NWA) }\end{array}$ & Broncita & $\left(\mathrm{Mg}_{0,786} \mathrm{Fe}_{0,236}\right) \mathrm{Si}_{0,980} \mathrm{O}_{3}$ \\
\hline $\begin{array}{l}\text { OAMC-5 } \\
\text { (NWA) }\end{array}$ & Broncita & $\left(\mathrm{Mg}_{0,787} \mathrm{Fe}_{0,198}\right) \mathrm{Si}_{0,991} \mathrm{O}_{3}$ \\
& Bnstatita & $\left(\mathrm{Mg}_{0,803} \mathrm{Fe}_{0,178}\right) \mathrm{Si}_{0,990} \mathrm{O}_{3}$ \\
& Broncita & $\left(\mathrm{Mg}_{0,799} \mathrm{Fe}_{0,191}\right) \mathrm{Si}_{0,993} \mathrm{O}_{3}$ \\
& Broncita & $\left(\mathrm{Mg}_{0,799} \mathrm{Fe}_{0,191}\right) \mathrm{Si}_{0,993} \mathrm{O}_{3}$ \\
& Augita & $\mathrm{Fe}_{0,798} \mathrm{Na}_{0,716} \mathrm{Al}_{0,801} \mathrm{Si}_{1,988} \mathrm{O}_{6}$ \\
\hline OAMC-6 & Broncita & $\left(\mathrm{Mg}_{0,802} \mathrm{Fe}_{0,186}\right) \mathrm{Si}_{0,993} \mathrm{O}_{3}$ \\
(NWA) & Pigeonita & $\left(\mathrm{Al}_{0,442} \mathrm{Na}_{0,341} \mathrm{Ca}_{0,465}\right)\left(\mathrm{Mg}_{0,327} \mathrm{Fe}_{0,125}\right)$ \\
\hline OAMC-7 & Broncita & $\left(\mathrm{Mg}_{0,729} \mathrm{Fe}_{0,205}\right) \mathrm{Si}_{0,964} \mathrm{O}_{3}$ \\
(NWA) & & \\
\hline
\end{tabular}

Tabla 7.-Composición de los óxidos presentes en las muestras de meteoritos

\begin{tabular}{cll}
\hline \multicolumn{1}{c}{ Meteorito } & Mineral & \multicolumn{1}{c}{ Fórmula } \\
\hline OAMC-5 (NWA) & Cromita & $\mathrm{Fe}_{0,876} \mathrm{Cr}_{1,596} \mathrm{O}_{4}$ \\
& Cromita & $\mathrm{Fe}_{0,854} \mathrm{Cr}_{1,633} \mathrm{O}_{4}$ \\
& Cromita & $\mathrm{Fe}_{0,835} \mathrm{Cr}_{1,607} \mathrm{O}_{4}$ \\
& Cromita & $\mathrm{Fe}_{0,860} \mathrm{Cr}_{1,614} \mathrm{O}_{4}$ \\
& Cromita & $\mathrm{Fe}_{0,820} \mathrm{Cr}_{1,631} \mathrm{O}_{4}$ \\
& Cromita & $\mathrm{Fe}_{0,866} \mathrm{Cr}_{1,635} \mathrm{O}_{4}$ \\
\hline OAMC-6 (NWA) & Magnetita & $\mathrm{Fe}_{3,738} \mathrm{O}_{4}$ \\
\hline
\end{tabular}

Tabla 8.-Composición de los sulfuros presentes en las muestras de meteoritos

\begin{tabular}{cll}
\hline \multicolumn{1}{c}{ Meteorito } & Mineral & \multicolumn{1}{c}{ Fórmula } \\
\hline OAMC-5 (NWA) & Troilita & $\mathrm{Fe}_{0,99} \mathrm{~S}_{1,01}$ \\
& & $\mathrm{Fe}_{0,99} \mathrm{~S}_{1,0}$ \\
& $\mathrm{Cr}_{0,01} \mathrm{Fe}_{0,99} \mathrm{~S}_{1,0}$ \\
& $\mathrm{Cr}_{0,01} \mathrm{Fe}_{0,99} \mathrm{~S}_{1,0}$ \\
\hline OAMC-6 (NWA) & Troilita & $\mathrm{Mg}_{0,03} \mathrm{Si}_{0,04} \mathrm{Fe}_{0,96} \mathrm{~S}_{0,96}$ \\
\hline OAMC-7 (NWA) & Troilita & $\mathrm{Ni}_{0,06} \mathrm{Fe}_{0,90} \mathrm{~S}_{1,04}$ \\
& & $\mathrm{Mg}_{0,05} \mathrm{Fe}_{0,94} \mathrm{~S}_{1,01}$ \\
\hline
\end{tabular}




\section{Conclusiones}

Los siete ejemplares estudiados del MCNT, pertenecen al grupo de las condritas ordinarias. La mineralogía se caracteriza por la presencia de olivino en general de naturaleza forsterítica, piroxeno de tipo enstatita y broncita, plagioclasa de composición albita-oligoclasa sólo identificada en uno de los ejemplares (OAMC-2 NWA). La presencia de metal libre (mayoritariamente kamacita) ha sido identificada en 3 ejemplares (OAMC-2 NWA, OAMC-5 NWA, OAMC-6 NWA). Los óxidos identificados son mayoritariamente cromita y menos magnetita. Los sulfuros identificados son del tipo troilita (OAMC-5 NWA, OAMC-6 NWA, OAMC-7 NWA).

Todos los datos obtenidos hasta el momento van completando las características descriptivas que permiten llevar a cabo la catalogación de los ejemplares así como ahondar en la investigación sobre los mismos, de cara a comprender su origen y las relaciones que existen entre los diferentes meteoritos. Uno de los objetivos fundamentales que supone la catalogación de las muestras, es su incorporación a la base de datos de la «Meteoritical Society», lo que constituye un hito museístico y científico que no tiene precedentes en España.

\section{AGRADECIMIENTOS}

Gracias al Museo de Ciencias Naturales de Tenerife (perteneciente al Organismo Autónomo de Museos y Centros del Cabil- do Insular de Tenerife), por su apoyo institucional y la cesión de los meteoritos para su investigación como parte de una tesis doctoral actualmente en curso en la Universidad de La Laguna.

\section{Referencias}

Grady, M.M. (2000). Catalogue of Meteorites: With Special Reference to Those Represented in the Collection of the Natural History Museum, London, 5th ed. Cambridge University Press, Cambridge, UK, $689 \mathrm{pp}$.

Hernández-Fernández, S.; Rodríguez-Losada, J.A.; García Talavera, F.; Lunar-Hernández, R. \& MartínezFrías, J. (2008). La colección de meteoritos del Museo de Ciencias Naturales de Tenerife: Catalogación y resultados preliminares. Geo-Temas, 10: 438.

Martínez-Frías, J.; García Guinea, J. \& Benito, R. (1989). Los Meteoritos. Mundo Científico, 93: 742-750.

Muñoz-Espadas, M.J.; Martínez-Frías, J.; Lunar, R.; Sánchez, B. \& Sánchez, J. (2002).The meteorite collection of the National Museum of Natural Sciences, Madrid, Spain: An updated catalog. Meteoritics \& Planetary Science, 37: B89-B94. doi:10.1111/j.19455100.2002.tb00907.x

Muñoz Sanz, J. (1997). Caracterizacion petrológica y geoquímica del meteorito «Valenciano». Tesis de Licenciatura, Universidad Complutense, Madrid, España. $96 \mathrm{pp}$.

Rull, F.; Martínez-Frías, J.; Sansano, A.; Medina, J. \& Edwards, H.G.M. (2004). A comparative micro-Raman study of Nakhla and Vaca Muerta meteorites. Journal of Raman Spectroscopy, 35: 497-503. doi:10.1002/jrs.1177

Recibido el 23 de diciembre de 2009 Aceptado el 18 de marzo de 2010 Publicado online el 9 de junio de 2010 